\title{
Brief Introduction of 1553B Bus Technology and Its Application Wei Huang ${ }^{1, a}$, Zhong Wang ${ }^{2, b}$ and Xin Wang ${ }^{3, c}$ \\ ${ }^{1,2,3}$ Xi'an Research Institute of High Technology, Xi'an, China

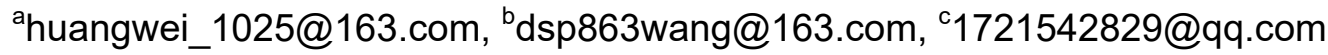

Keywords: 1553B bus; reliability; message transmission format; application

\begin{abstract}
B bus with its reliability and real-time plays an important role in the field of military data bus. This paper introduces the history, characteristics and message transmission format of 1553B bus, analyzes the reliability of 1553B bus communication system, compares several typical buses, and discusses the application of 1553B bus in weapon system.
\end{abstract}

\section{Introduction}

With the development of computer technology, communication technology and integrated circuit technology, a variety of bus standards came into being. 1553B (MIL-STD-1553B) is a serial multiplex data bus standard published in 1978 by the United States among them. It's a military standard on the data bus electrical characteristics and communication protocol specification. It specifies the technical requirements of the digital command / response time multiplexed data bus within the aircraft, as well as the operation of the multiplexed data bus, the format of the information flow on the bus, and electrical requirements, and provides the medium to transfer data and information between different systems.

As the first generation of military data bus, 1553B bus was originally proposed for the fighter's internal electronic system networking standards, with its high reliability, speed, proven technique, easy to expand and other characteristics, and it has been gradually extended from the flight control systems to missiles, ships, aerospace and other fields, after 30 years without failure. It's known as a net cover of the armed forces. In recent years, 1553B bus has become the most important advanced avionics system data bus in China's active air force, and it has played an important role in the field of ships and vehicles, tanks and warships and other weapons to improve the technical level ${ }^{[1]}$.

\section{Introduction of 1553B Bus}

\section{B bus development history.}

In the 1960s, third-generation fighters had an integrated fire control system consisting of inertial, satellite navigation / head-up display / weapon aiming systems and long-range air-launched weapons. Its combat capability greatly improved. At the same time, the total amount of operational information data has increased rapidly, and there are differences between the interface of systems and devices, interconnection difficult, unable to further enhance the combat efficiency, and it's so difficult to interconnect that can't further enhance combat effectiveness. And the cost of the development, maintenance and improvement increases constantly because there is no uniform standard.

To solve these problems, the US SAE's AE-9E Committee decided to develop a standard signal multiple transmission system in 1968, and announced the MIL-STD-1553 (USAF) standard in 1973, and for the first time used this standard in the F-16 fighters. Thus it introduced bus technology so that avionics devices cross-linked through the bus. The difference between the independent avionics and avionics of bus structure is shown in Fig.1 and Fig.2. 


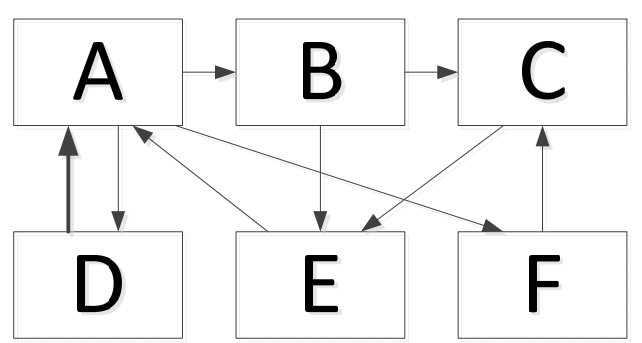

Fig. 1 Cross-linked schematic diagram of independent avionics system

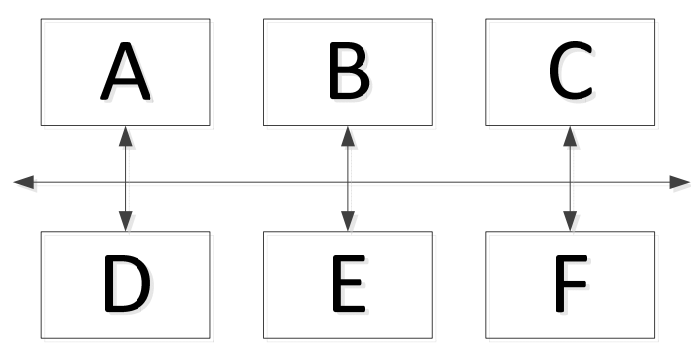

Fig. 2 Cross-linked schematic diagram of avionics system of bus structure

With the improvement of technology, improved MIL-STD-1553A was announced in 1975 which was still used in the United States Air Force F-16 fighters and "Apache" new attack helicopter AH-64A. The MIL-STD-1553B standard was also announced in 1978. After 1980, the US Air Force has made improvements to this standard, and 1553 Notice 1 was introduced. The Notice 2 was introduced in 1986 due to many restrictions in Notice 1. But the standard is still dominated 1553B, the standard has been used up to now.

1553B standard is similar to the communication protocol. It replaces the huge equipment which is used to transfer data between the sensor, computer, indicator and other aircraft equipment, improves electromagnetic compatibility, reliability and fault tolerance of intelligence equipment, and has become an integral part of avionics systems which can be used as modern combat aircraft electronic system "brain center".

1553B bus characteristics.

1553B bus advantages exist in reducing the size, weight, complexity and cost of electronic systems integrated electronic devices, etc. It has become the main pillars of work of a mobile platform electronic system, and has distinctive characteristics different from the general electronic network.

(1) 1553B bus is a computer networks of broadcast distribution processing, which can be connected 32 terminals. All terminals (nodes) share a single message path, and at any one time only one can send a message in network. The messages in transit can be received by all terminals, and the actually received terminals are identified by addresses. The network structure is simple, and it's convenient to expand. In addition to the bus controller, the failure of any terminal will not cause the entire network paralysis, and the bus controller can be backed up to improve reliability. The network is more sensitive to the failure of the bus itself, so it usually use dual-redundancy bus.

(2) Real-time. 1553B bus has a transfer rate of $1 \mathrm{Mbps}$ and contains up to 32 words per message (16 bits per word), so the time to transmit a message is relatively short.

(3) 1553B bus in accordance with the command / response mode asynchronous operation, that is, all messages transmission on the bus is controlled by the instruction issued by bus controller, and the relevant terminal shall respond to the instruction and perform the operation. This approach is ideal for centralized control of distributed processing systems.

(4) Taking into account the real-time conditions, it uses reasonable error control measures, which is the feedback retransmission method, and enhances the reliability of communication ${ }^{[2]}$.

\section{B Bus Technology}

\section{B Bus Communication System.}

From the perspective of the communication system, one of all subsystems acts as a bus controller (BC), and the other subsystems are remote terminals (RT). 1553B bus can be connected to 31 remote terminals, complete the communication and data exchange of the subsystems, and realize the centralized control and display of the subsystems. In order to improve reliability, 1553B bus generally dual redundant structure. System structure redundancy example is shown in Fig.3. 


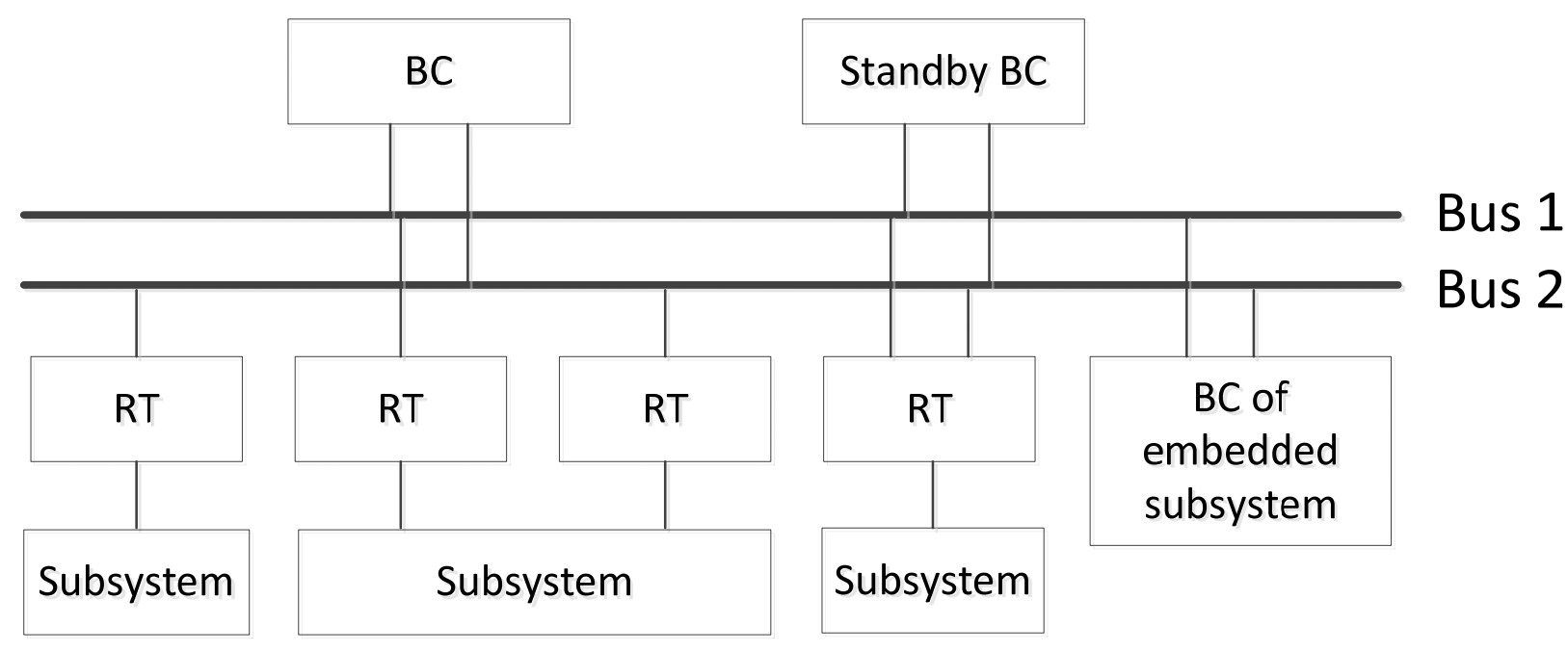

Fig.3 system structure redundancy example

\section{B Bus Message Transmission Format.}

$1553 \mathrm{~B}$ bus information is modulated in the form of information into Manchester II code for transmission. Manchester II type code is a two-phase level code, encoding shown in Fig.4. Logic 1 is the bipolar coded signal 1/0 (ie, one positive pulse followed by one negative pulse). Logic 0 is the bipolar coded signal $0 / 1$ (ie, 1 negative pulse followed by 1 positive pulse). The zero-crossing transition occurs at the midpoint of each bit.

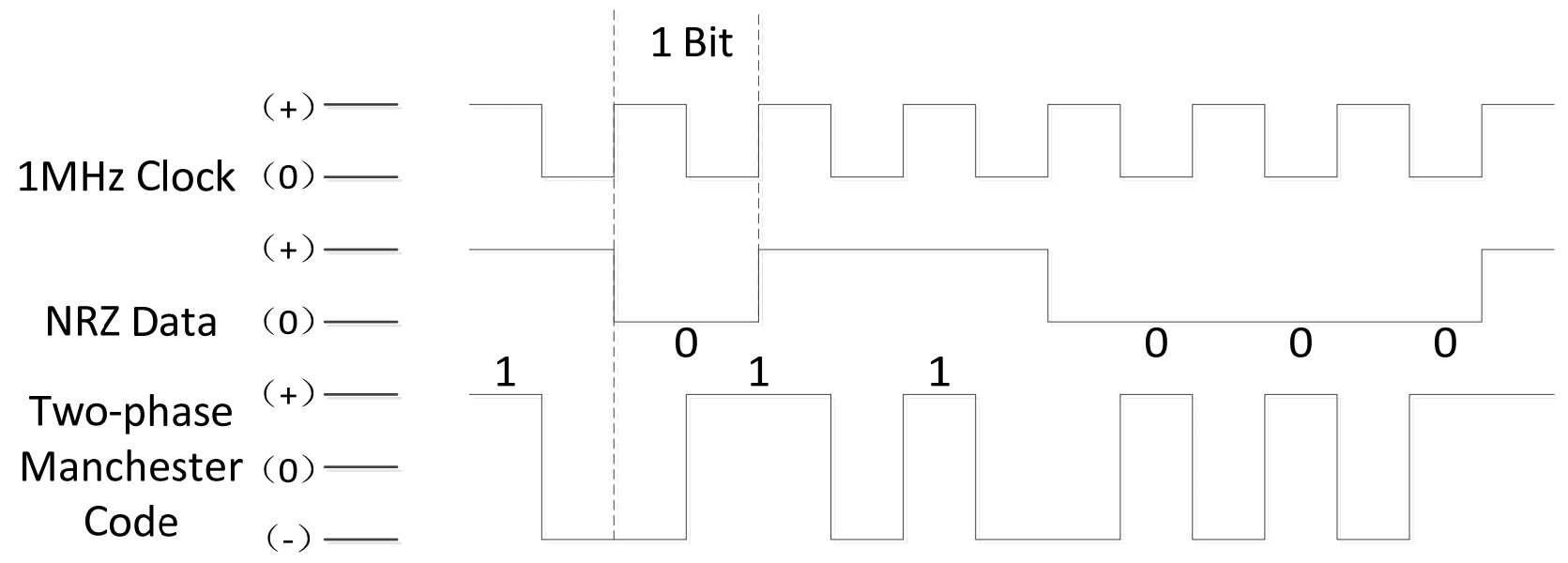

Fig.4 coding

\section{1) 1553B Word Format}

1553B information flow consists of a series of 1553B messages, each message up to 32 words, and all words are divided into three categories: the command word, data word and status word. These three words are 3-bit synchronous header +16 -bit valid bit +1 bit parity bit, a total of 20 bits. The sync header and parity bits are used when determining message format and data errors. The word format is shown in Fig.5. 
Bit

$$
\begin{array}{|l|l|l|l|l|l|l|l|l|l|l|l|l|l|l|l|l|l|l|l|}
1 & 2 & 3 & 4 & 5 & 6 & 7 & 8 & 9 & 10 & 11 & 12 & 13 & 14 & 15 & 16 & 17 & 18 & 19 & 20 \\
\hline
\end{array}
$$

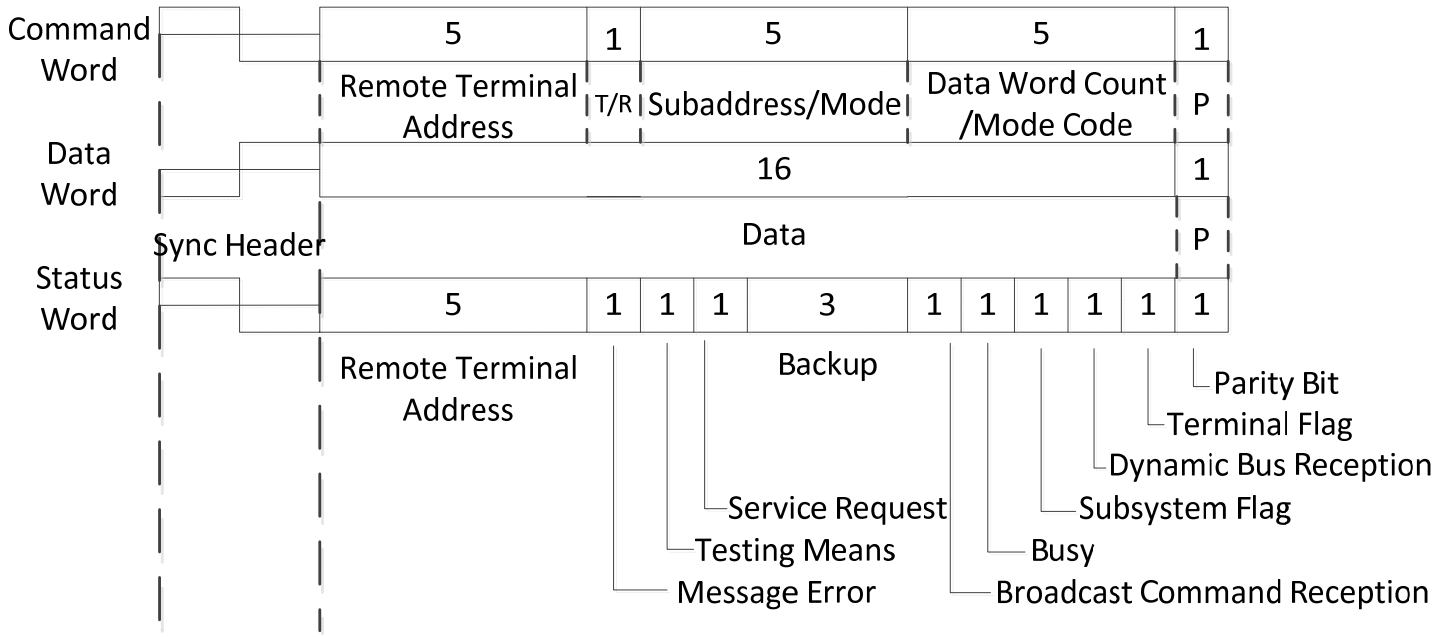

Fig.5 word format

\section{2) 1553B Message Formats}

In the 1553B bus protocol, it defines 10 message formats, as shown in Fig.6.

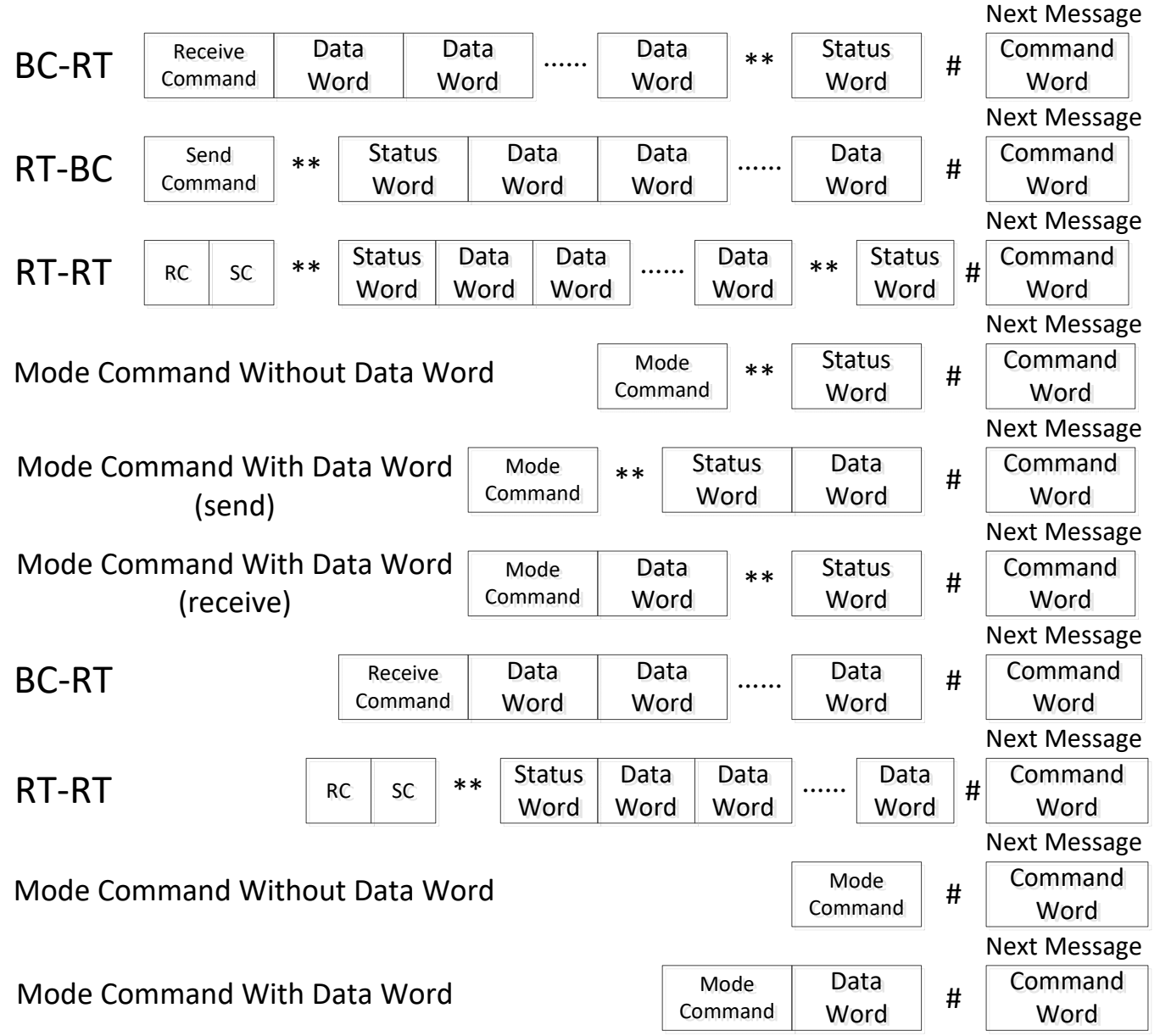

Fig.6 message format ( ${ }^{* *}$ indicates the response time, \# indicates the message interval)

The first six is information transmission format, and the latter four is broadcast information transmission format. Information transfer must be directly controlled by $\mathrm{BC}, \mathrm{RT}$ being accessed to make a specific response; Both $\mathrm{BC}$ and RT can send messages when they are broadcast. The efficiency of broadcast transmission is high, but the message sender can't confirm the receiving end of the message acceptance status. It's difficult to guarantee the reliability of transmission, so the use 
of broadcast message format must be cautious.

\section{3) 1553B Mode Instruction}

Mode instructions are specifically designed for BC communications or the monitoring, diagnostics and control of terminal error faults. When the sub-address in the instruction word sent by the $\mathrm{BC}$ is all 0 or all 1, the instruction is a mode instruction. But the specific mode instruction is represented by the 5-digit code in the digital address. Mode codes of mode instruction are the following: Dynamic Bus Control (00000); Synchronization (00001); Sending the last status word (00010); Starting self-test (00011); Transmitter off (00100); Canceling Transmitter Off (00101); Prohibiting remote terminal flag (00110); Canceling Prohibiting remote terminal flag (00111); Resetting the remote terminal (01000); Sending the vector word (10000); Synchronization without data words (10001); Synchronization with data words (10010); Sending word detection word (10011); The selected transmitter off (10100); Canceling the selected transmitter off $(10101)^{[3]}$;

\section{Comparison of Several Typical Bus}

There are a number of open LAN standards are widely used in the commercial field. WAN and MAN have strong global capabilities, but they are not suitable for use as backbone networks for aircraft and vehicles. Only LAN can be used as a candidate backbone network technology, including: Fiber Channel, Fiber Distributed Data Interface (FDDI), Ethernet, FireWire / IEEE 1394, Asynchronous Transfer Mode (ATM) and Universal Serial Bus (USB) etc.

When comparing different technologies, their application and maturity are as important as their technical comparisons. Some technologies such as Serial Express, 1 Gbps Enet, USB, Fiber Channel, ATM, IEEE 1394, CAN bus, 100 Mbps Enet, 10 Mbps Enet, Utility Bus, FDDI, F-22 HSDB are in the state of being introduced and eliminated. Cutting-edge technology is not mature enough, so it is not widely used in military systems. Others, although mature, are declining if applied to modern new systems.

This paper does not consider the current emerging interconnection standards, mainly because the networking technology on the weapon platform is different from the general LAN technology. It emphasizes the network reliability, real-time and adaptability to the harsh environment. Now there are competitive backbone network technology has the following: FDDI, Fiber Channel Arbitrated Loop (FC-AL), ATM ${ }^{[4]}$. Table 1 compares these techniques with the 1553B.

Table 1 bus comparison

\begin{tabular}{|c|c|c|c|c|}
\hline Item & 1553B & FDDI & FC-AL & ATM \\
\hline Topology & Linear bus & Token ring & Arbitration ring & Hub-and-spoke \\
\hline $\begin{array}{l}\text { Deterministic } \\
\text { transmission }\end{array}$ & Yes & No & Yes & No \\
\hline Operation & Asynchronous & $\begin{array}{c}\text { Asynchronous or } \\
\text { Synchronous }\end{array}$ & $\begin{array}{c}\text { Asynchronous or } \\
\text { Synchronous }\end{array}$ & \\
\hline $\begin{array}{c}\text { Temperature span } \\
{\left[{ }^{\circ} \mathrm{C}\right]}\end{array}$ & 180 & 130 & 145 & 70 \\
\hline $\begin{array}{c}\text { Transmission } \\
\text { technology }\end{array}$ & Half duplex & & Full or Half duplex & \\
\hline Number of nodes & 32 & 16 & 16 & 16 \\
\hline $\begin{array}{c}\text { Transmission rate } \\
{[\mathrm{Mbit} / \mathrm{s}]}\end{array}$ & 1 & 100 & 100 & 155 \\
\hline Encoding & Manchester & NRZ-I & $8 \mathrm{~B} / 10 \mathrm{~B}$ & \\
\hline $\begin{array}{l}\text { Data delay } \\
\text { (Worst case) }\end{array}$ & 60 & 36 & 30 & 300 \\
\hline $\begin{array}{c}\text { Initialization time } \\
{[\mathrm{ms}]}\end{array}$ & 0.1 & 10 & 1 & 10 \\
\hline $\begin{array}{c}\text { Degree of } \\
\text { real-time control }\end{array}$ & 10 & 6 & 5 & 1 \\
\hline Application & All & Navy & Air Force & No \\
\hline Maturity & 10 & 6 & 3 & 3 \\
\hline
\end{tabular}


As can be seen from Table 1, FDDI is a more mature backbone network technology, and it's also used in the military field, but it has not been widely accepted. Although it is inherently redundant, there is no good real-time deterministic data transmission scheme. Compared with other technologies, the maximum data transfer rate is slower.

FC-AL is a relatively advanced technology, but it's not yet mature and widely used. From the military point of view, it also need time to test.

ATM as an emerging technology is supported in the telecommunications switch market, but it is not mature enough, and its upgrade space is small, and it can't guarantee real-time transmission of data. In addition, its communication protocol is not reliable enough and the operating temperature range is small, so it is not able to adapt to harsh environments.

1553B bus is the application of the earliest and most durable in the military data bus. Because it is put forward to meet the needs of industrial and military defense, it has a high reliability and flexibility. The United States F-22 fighter still uses 1553B data bus as the control bus, and it is used with token-based fiber optic transmission bus SAE4074, so its importance is self-evident.

From the above comparison, it is clear that the 1553B technology is very mature for data lines requiring high bandwidth and low latency, and is widely used in the military field ${ }^{[5]}$.

\section{Application of 1553B Bus}

Modern weapons on communications systems generally have the following requirements: (1) To meet specific communication characteristics, the realization of the data transmission between subsystems; (2) Communication subsystems work relatively independently, and it is as transparent as possible to application software, and it takes up the host of time as little as possible; (3) The communication subsystem is designed to be flexible and easy to modify; (4) The communication subsystem has strong anti-interference ability.

$1553 \mathrm{~B}$ bus meets the above requirements exactly, so that it is more and more widely used in the modern weapon system. Since the United States announced the military standard MIL-STD-1553 in 1973, it's quickly applied to the F-16, F-18 and F-22 and other fighters.

Now available military data bus a lot, but $1553 \mathrm{~B}$ bus is the most widely used. 1553B bus transmission medium has coaxial cable, shielded twisted pair and fiber optic cable etc., and it couples the terminal to the bus via transformer coupling or direct coupling. Transfer rate, transmission distance and the number of remote terminals of 1553B bus are in line with a variety of small and medium-sized ships and submarine system communication requirements, so it have also been widely used in the naval ships.

As the Army ground weapons, all types of tanks often work in harsh environments with strong vibration, noisy, dusty and large temperature variations, so the fault detection of data communication between internal electronic devices must be very strict. In order to control the engine and the transmission in real time, the data communication between them requires the maximum response time of a message as short as possible, so as to improve the overall performance of the whole power system. 1553B bus has high reliability and good real-time, and it has more performance advantages than the vast majority of the existing bus for the system with the integrated control of power transmission of this type of data communications, large amount of data, real-time requirements, and less network nodes ${ }^{[6]}$.

\section{Summary}

As an important military data bus, 1553B bus has a high transmission rate, good environmental adaptability, electromagnetic compatibility and redundancy fault tolerance. 1553B bus adopts dual redundant structure to ensure a good fault tolerance and fault isolation; The Manchester encoding format adopted in the protocol guarantees the wrong detection position and wrong isolation; Word format and message transmission format ensure the error correction and detection of positioning; The transmission medium of the bus adopts shielded twisted pair, and it adopts the way of coupling to connect with the terminal effectively restraining the external electromagnetic interference, so it 
has guaranteed the reliability of communication. Therefore, 1553B bus not only now occupies an important position in the military field, and in the future for a long time will continue to be applied.

\section{References}

[1] Xu Guixian .1553B bus profile and its implementation [J]. Communications Technology, 2011, 05: 166-168.

[2] Liu Shiquan, Jun Yang, Cai Jieming, Wei Jinghe, Huang Zheng.Application and Development of 1553B Bus [J]. Electronics \& Packaging, 2013, 12: 12-15.

[3] Huang Jiaping, Wang Minghao, Xie Huiling, Lv Zhaohui .1553B bus communication system reliability analysis $[\mathrm{J}]$ aircraft design, 2010,02: 57-61.

[4] Yang Liu. On the 1553B bus and its application development [J]. Friends of Science, 2012, 08: 159-161.

[5] Ma Guibin, Zhou Guochi, Tian Ke.Multi-level Development of Military Data Bus Technology [J] .Photo Optics \& Control, 2010,06: 48-53.

[6] $\mathrm{Hu}$ Laihong, Xu Hualong. Application of 1553B bus in weapon communication [J]. Microcomputer Information, 2005, 20: 56-57 +124. 